\title{
Applications \\ de la méthode de l'hydrogramme unitaire à quelques cours d'eau français
}

\section{Application of the unit hydrograph method to a few French streams}

\author{
PAR J. JACQUET
}

INGÉNIEUR A LA DIVISION « HYDROLOGIE》

DU SERVICE DES ÉTUDES FrYDRAUI.IQUES D'ÉLECTRIGITÉ DE FRANGE

\begin{abstract}
Examen des problèmes d'hydrologie -- relatifs d l'analyse et à la reconstitution des débits résultant d'ane averse, $\dot{a}$ leur distribution dans le temps -, susceptibles d'être étudiés par la méthode de l'hydrogramme unitaire.

Presentation des résultats obtenus et indications sur les etudes en cours relatives aux conditions d'application de cette méthode à certains cours d'eau français dont les superficies de bassins versants varient de $3 \dot{a} 600 \mathrm{~km}^{2}$. Questions pratiques de mise en cuvre de la méthode d'après quelques exemples (analyse d'averses et des crues correspondantes pour la dérivation d'hydrogrammes unitaires, synthèse d'hydrogrammes, etc.).

Intérét et limites d'application de cette technique.
\end{abstract}

Les principes de base de la méthode de l'hydrogramme unitaire étant connus [1], on examine dans une première partie les problèmes concrets de l'ingénieur qui justifient la mise en œuvre pratique de cette technique; les seconde et

\begin{abstract}
Examination of hydrological problems likely to be studied by the unit hydrograph method, in connection with the analysis and reconstitution of flows resulting from a shower and their distribution with respect to time. Presentation of results obtained and indications regarding present studies of conditions under which this method can be applied to certain French streams, the catchment areas of which vary between $3 \mathrm{lnm}^{\circ}$ and $600 \mathrm{~km}^{2}$.

Practical application questions shown by a few examples (analysis of showers and resulting floods in order to determine unit hydrographs, synthesis of hydrographs, etc.).

Advantages and limits of application of the method.
\end{abstract}

troisième parties de cet exposé sont consacrées à une revue rapide de résultats d'application à l'analyse des phénomènes d'écoulement sur divers cours d'eau de France. 


\section{CONDITIONS PRATIQUES D'UTILISATION DE LA MÉTHODE DE L'HYDROGRAMME UNITAIRE}

Le problème fondamental cnvisagé ici est celui de la synthèse de l'hydrogramme d'écoulement d'une averse sur un bassin versant : dans quelle mesure l'utilisation d'hydrogrammes unitaires en permet-elle une approche de solution? On est conduit à distinguer divers points de vue suivant la nature du résultat recherché par l'ingénieur et suivant le type de bassin versant étudié.

\section{A. - Problèmes hydrologiques d'équipement et d'exploitation des cours d'eau}

En matière d'hydrologie des crues, l'ingénieur d'équipement et l'ingénieur exploitant d'aménagements hydrauliques ont des exigences différentes, bien qu'elles se ramènent en dernière analyse à la recherche d'informations quantitatives sur des phénomènes à venir, à espérer ou à craindre, avec ou sans indication de temps.

1. La détermination des dimensions d'un ouvrage nécessite la connaissance des caractérisliques des crues de faibles probabilités au point d'implantation choisi : distribution des débits dans le temps et valeur du débit de pointe de l'hydrogramme de crue, sans référence - et c'est important - à une date précise d'occurrence du phénomène. Il sera en principe avantageux d'utiliser la méthode de l'hydrogramme unitaire lorsque la statistique des débits dont on dispose est courte au regard de celle des données pluviométriques. Mais il faut noter que l'introduction du point de vue probabiliste dans la liaison pluie-débit à l'échelle de l'averse, pose des problèmes délicats qui sont loin d'avoir reçu des solutions satisfaisantes.

2. Les exigences d'un organisme d'exploitation sont plus précises et aussi plus difficiles à satisfaire: il s'agit en effet de prévoir un hydrogramme d'écoulement à sa date exacte, à échéance la plus longue possible (annonce des crues, pré- vision d'apports dans une retenue, etc.). L'hydrogramme unitaire, en tant que caractéristique du bassin versant, est utilisé à son rang pour moduler dans le temps le volume d'écoulement estimé par d'autres néthodes à partir des averses observées ou prévues : il n'apporte donc pas une solution complète à ce prohlème complexe.

\section{B. - Prédétermination d'hydrogrammes d'écoulement d'une aire imperméabilisée et $d$ 'un bassin versant naturel}

1. La presque totalité des eaux météoriques reçues par une agglomération à forte densité de population contribue au ruissellement superficiel direct. Les coefficients d'écoulement ou de ruissellement sont toujours élevés et dépendent d'abord du degré d'imperméabilisation, donc d'urbanisation, du bassin. On utilise en pratique des valeurs fixes de ces coefficients, caractéristiques de l'aire étudiée. Ceci représente une simplification importante du calcul des crues pluviales à évacuer, qui ne comportent par ailleurs qu'un débit de base négligeable. Si des hydrogrammes unitaires ont pu être déduits de l'analyse d'un certain nombre d'averses et de crues subsćquentes observées sur la zone, la détermination des débits maximaux correspondant à des averses-types de fréquence déterminée est, en principe, possible.

2. La difficulté de prédéterminer les hydrogrammes de crues d'un bassin versant naturel, dont on connait des hydrogrammes unitaires, provient de ce que les paramètres représentatifs de l'ètat du bassin varient rapidement dans le temps. Les valeurs des coefficients d'écoulement et de ruissellement d'averses dépendent étroitement de l'humidité des terrains, de l'état des nappes phréatiques sur le bassin, tout autant que des caractéristiques des précipitations. La distribution dans le temps _- en cours d'averse -. des «pertes» de précipitations («pertes » par infiltration, évaporation, rétention) pose enfin uu 
problème ardu non résolu. La complexité des facteurs en jeu nécessite donc l'examen d'un grand nombre de cas pour réaliser le « toutes choses égales d'ailleurs » dans l'étude du sens et de l'importance de l'influence de chaque facteur d'écoulement sur les hydrogrammes de crues.
C'est là l'objeć principal des études sur bassins expérimentaux qui permettent de transposer certains résultats à d'autres hassins, ou tout au moins d'orienter efficacenent les recherches sur un nombre limité de facteurs d'écoulement pour la prédétermination des hydrogrammes.

\section{DEUXIEME PARTIE}

\section{ETUDES EN COURS RELATIVES AUX CONDITIONS D'APPLICATION DE LA METHODE DE L'HYDROGRAMME UNITAIRE A DES COURS D'EAU FRANÇAIS}

Dans leur phase actuelle, ces études concernent l'analyse de crues d'origine pluviale sur des bassins versants naturels du Massif Central et de sa périphérie (fig. 1).

\section{A. - Etudes sur bassins expérimentaux}

1. Une vérification expérimentale de la validité des hypothèses de base de la méthode a ćté effectuée par M. Cappus [2] qui a analysé plus de 200 crues pluviales de la Saignerie sur le bassin expérimental d'Alrance (superficie $3,15 \mathrm{~km}^{2}$ ). Cet important travail a permis de dégager, pour les différentes phases d'écoulement, des relations permettant de les définir quantitativement et de suivre leur évolution dans le temps : il est

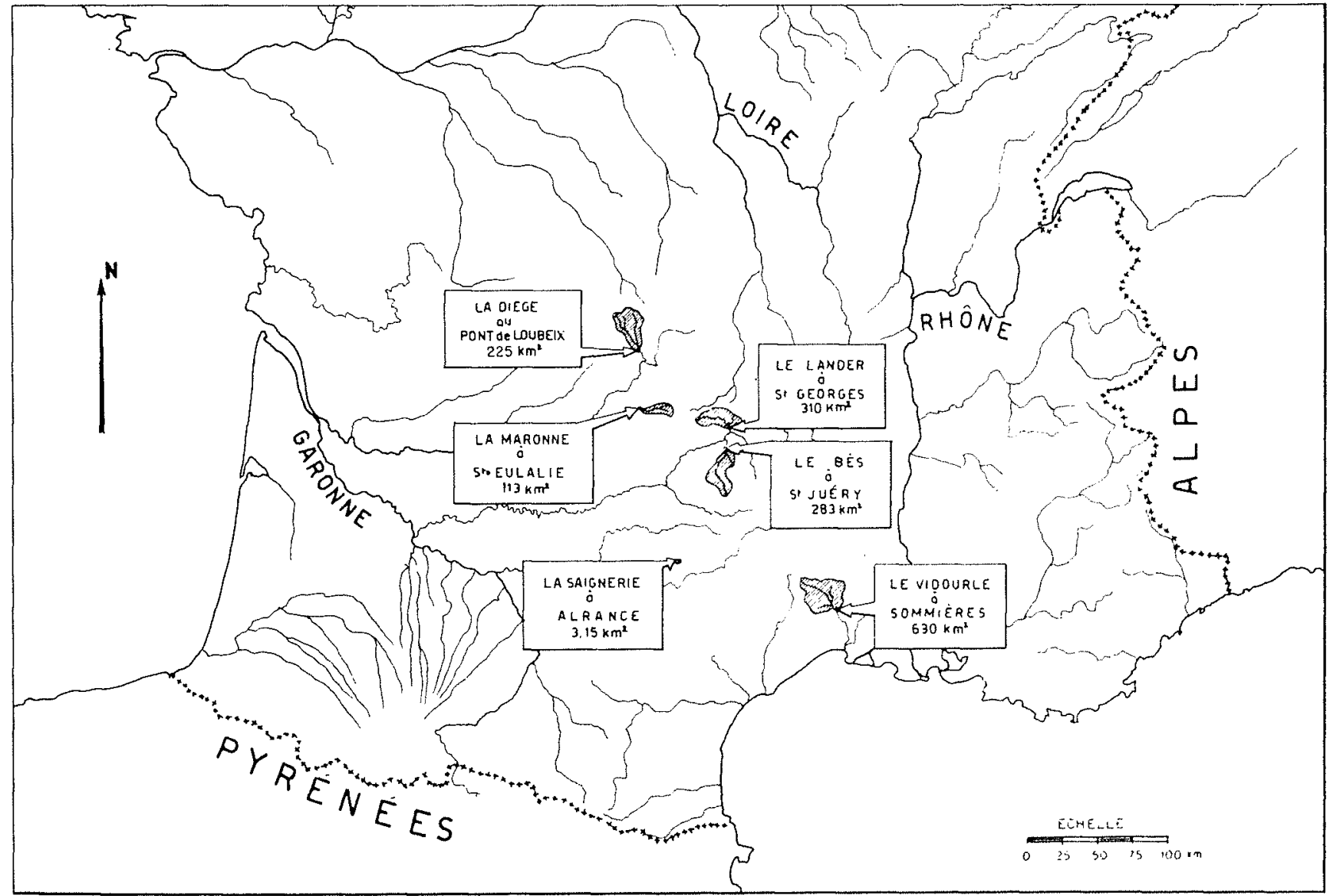

Fig. 1

Emplacement des bassins versants etudiés. 


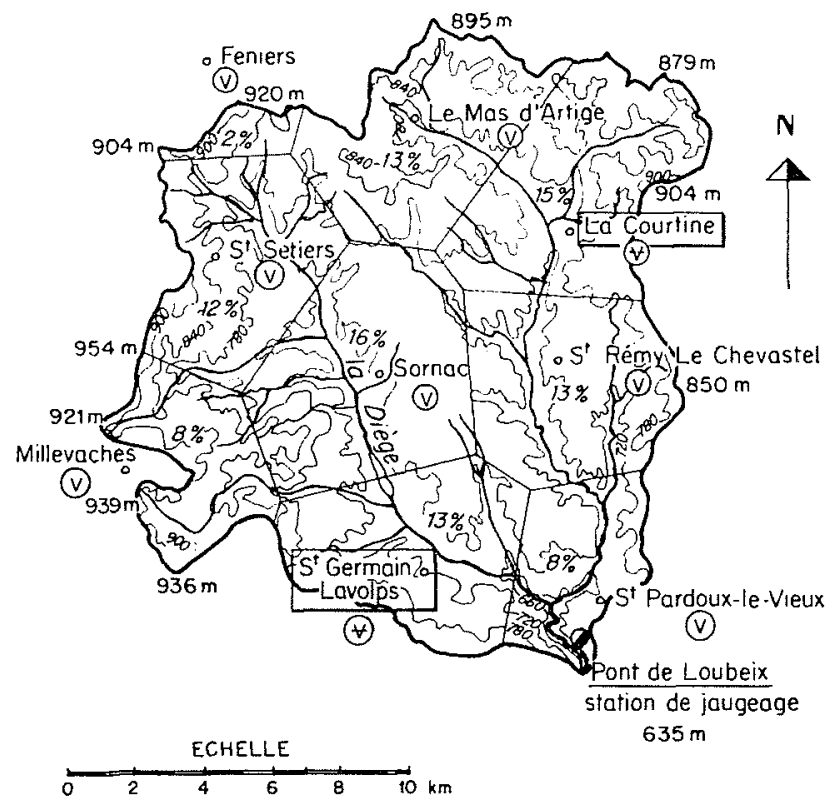

( Station pluviométrique équipée d'un pluviographe

$$
\begin{gathered}
\text { (v) " } " \text { "pluviomètre simple } \\
\text { FIG. } 2
\end{gathered}
$$

Bassin versant de la Diège au pont de Loubeix (superficie $225 \mathrm{~km}^{2}$ )

(On a représenté le découpage polygonal définissant les coefficients de pondération à affecter aux observations pluviométriques pour le calcul de la lame d'eau moyenne précipitée sur le bassin par la méthode de Thiessen.)

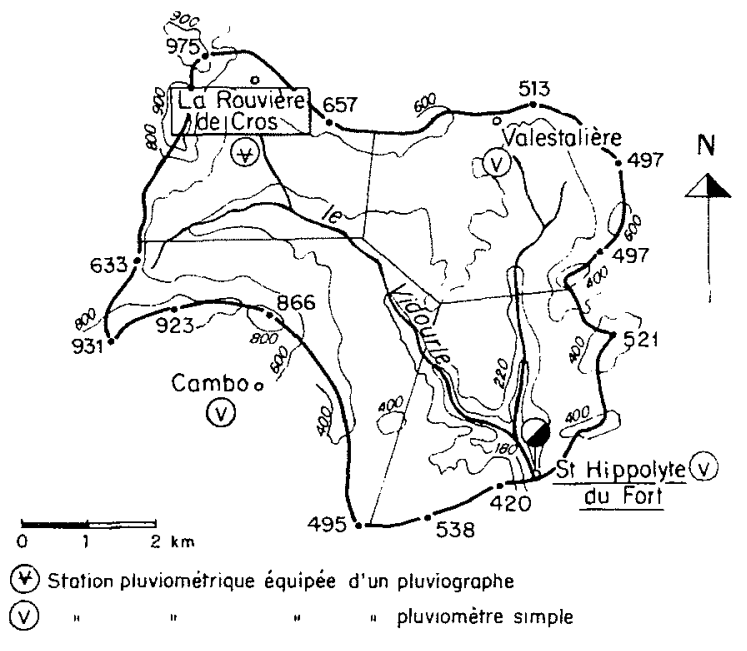

Fig. 3

Bassin versant du haut Vidourle

à Saint-Hippolyte-du-Fort (superficie : $35,5 \mathrm{~km}^{2}$ ).

possible de calculer avec une bonne précision les débits instantanés, moyens journaliers ou mensuels à partir des précipitations et d'un petit nombre de paramètres représentant l'état du bassin.

2. Une nouvelle phase de recherches en découle : contròler la validité du procédé de cal-

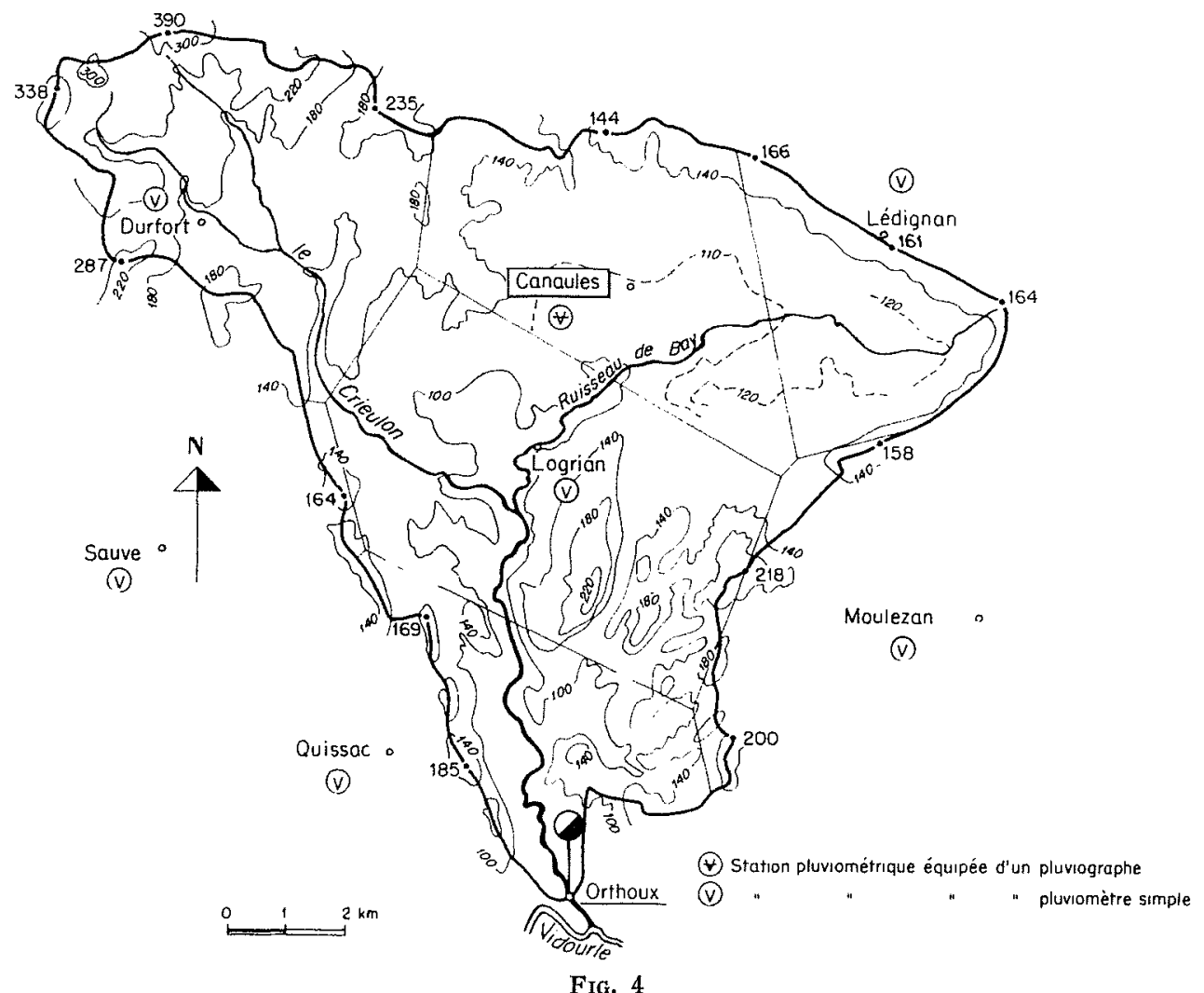

Bassin versant du Crieulon au pont d'Orthoux (superficie: $110 \mathrm{~km}^{2}$ )

(Lc Crieulon est un affluent de rive gauche du Vidourle à l'amont de Sommières.) 
cul mis au point à Alrance lorsqu'on l'applique à des bassins versants plus étendus et étudier l'évolution de la précision d'estimation des débits lorsque l'on s'écarte des conditions expérimentales d'établissement des relations pluic-débit dont on fait usage. La nécessité d'un ajustement qui prenne en compte les hétérogénéités croissantes affectant les facteurs d'écoulement est en effet évidente. A cette fin, une série de bassins versants expérimentaux dont les superficies varient de 20 à $225 \mathrm{~km}^{2}$ - tel celui de la Diège (fig. 2) - ont été équipés sur la haute Dordogne en vue de l'étude des possibilités de prévision d'apports à courte et moyenne échéance dans les retenues de la région.

\section{B. - Application de la méthode de l'hydro- gramme unitaire à l'étude des crues du Vidourle}

Cette étude, entreprise à la demande de la Compagnie Nationale d'Aménagement du Bas-Rhône et du Languedoc, avait pour objet la recherche de caractéristiques des crues de ce fleuve et de certains de ces affluents, en divers points où est prévue l'implantation de bar'rages écrêteurs pour la protection des localités riveraines. Les crues exceptionnelles de l'automne 1958 [3] ont per- mis de vérifier la stabilité des hydrogrammes unitaires en tant que caractéristiques de ruisscllement d'un bassin versant : les hydrogrammes unitaires dérivés de l'analyse de crues de moindre importance ont été utilisés pour reconstituer avec une précision acceptable les hydrogrammes de ces ciues de fréquence rare, sur le haut Vidourle à Saint-Hippolyte-du-Fort (fis. 3), sur le Crieulon à Orthoux (fig. 4) et sur le Vidourle à Sommières.

\section{Etudes sommaires sur des bassins du Massif Central}

Utilisant l'information brute dont on dispose sur des bassins versants équipés au moins d'un pluviographe et d'une station de jaugeage avec limnigraphe, on a pu montrer que l'analyse d'un nombre relativement restreint de crues simples (5 à 15 par bassin) permettait de dégager des renseignements utilisables pour la recherche de liaisons pluie-débit : des hydrogrammes d'averses types ont été tracés pour le Bès et le Lander, affluents de la Truyère (fig. 1) qui débouchent dans la relenue de Grandval, el pour la Maronne à Sainte-Eulalie (fig. 5), à l'amont du barrage d'Enchanet.

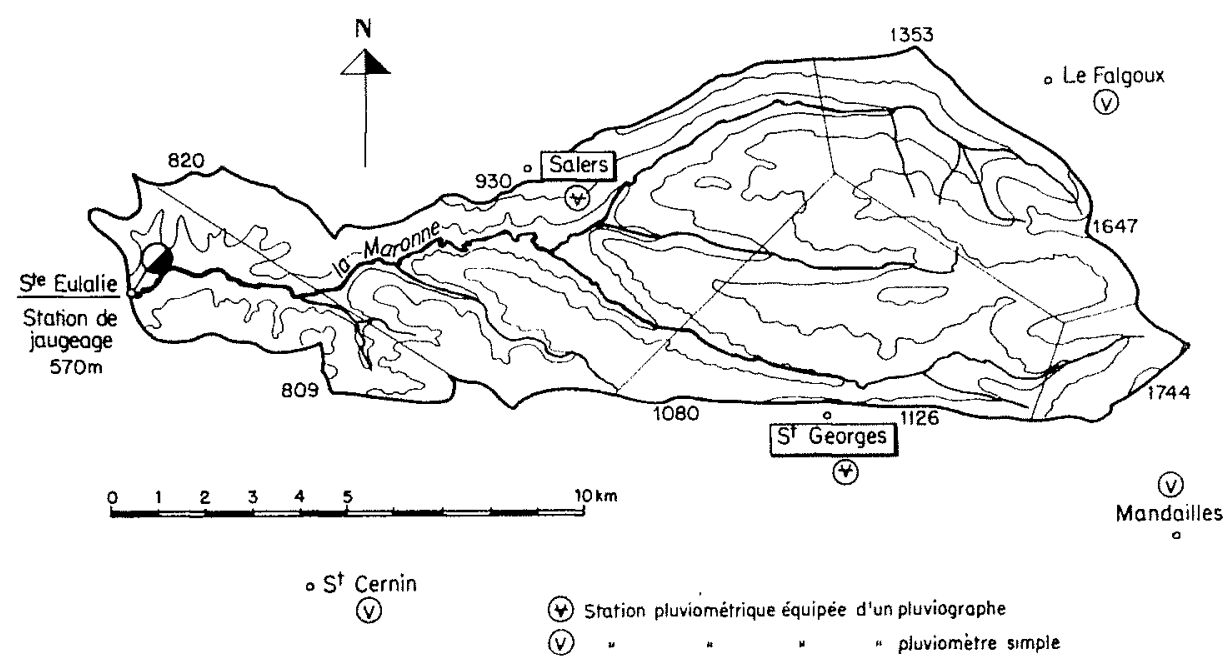

Fig. 5

Bassin versant de la Maronne a Sainte-Eulalie (superficie: $113 \mathrm{~km}^{2}$ ). 


\section{EXAMEN, SUR QUELQUES EXEMPLES, DES PROBLEMES PRATIQUES D'APPLICATION DE LA MÉTHODE DE L'HYDROGRAMME UNITAIRE}

Les problèmes (résolus ou non) que pose l'analyse des hiétogrammes d'averses et des hydrogrammes de crue en vue de la dérivation d'hydrogrammes unitaires, et ceux relatifs à la synthèse d'hydrogrammes d'écoulement à partir de la pluie, sont examinés à la lumière des résultats obtenus sur les divers bassins précités. Les définitions de termes techniques et les notations utilisées sont celles de L'Hydrologie de l'Ingénieur, de M. Rementeras [1].

\section{A. - Analyse des averses et des crues correspondantes pour l'établissement d'hydrogrammes unitaires}

1. Etude de L'Averse génératrice de ta chue (crue du Vidourle à Sommières, le 4 octobre 1958).

a) Estimation de la lame d'eau moyenne recue par le bassin :

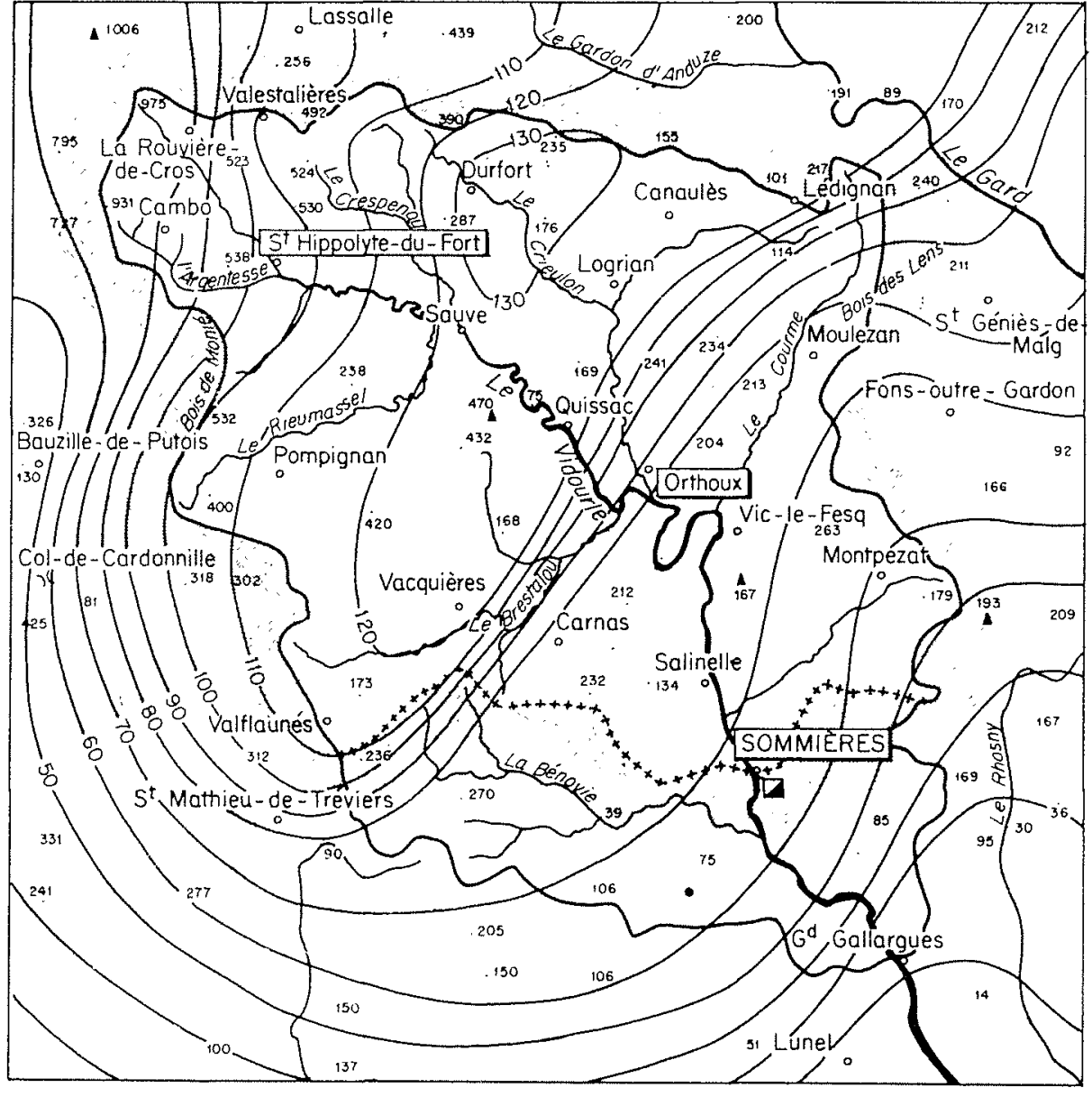

FIG. 6

Réseau d'isanomales cotées en \% de la hauteur de lame d'eau moyenne $(\mathrm{P}=153 \mathrm{~mm})$ précipitée sur le bassin du Vidourle à Sommières le 4 octobre 1958 (superficie du bassin versant : $630 \mathrm{~km}^{2}$ ). 
Cette estimation résulte d'une moyenne pondérée des hauteurs de précipitations observées aux divers pluviomètres du bassin et de sa périphérie d'après la méthode de Thiessen [1]. Les poids étant uniquement fonction de la répartilion topographique des stations, des erreurs systématiques peuvent s'introduire lorsque, par exemple, les pluviomètres sont tous situés dans les parties basses d'un bassin de montagne. Il importe donc de s'assurer que l'échantillonnage d'observations pluviométriques représente convenablement les précipitations réelles. Avec un réseau de stations tel que celui dont on dispose sur le bassin de Vidourle [3] ou sur celui de la Diège (fig. 2), l'écart entre les estimations de lame d'eau moyenne obtenues par la méthode de Thiessen et par planimétrage des isohyètes est inférieur à $5 \%$. Il est toutefois nécessaire de tracer des isohyètes ou isanomales d'averses pour étudier la répartition spatiale des précipitations qui, dans le cas présent, sont bien centrées sur le bassin (fig. 6).

b) Détermination du hyétogramme moyen d'averse sur le bassin :

L'hétérogénéité de répartition spatiale et temporelle de la pluie nécessite généralement l'installation de plusieurs pluviographes sur le bassin étudié. On calculera donc un hyétogramme moyen à partir d'une moyenne, pondérée ou non, des pourcentages de la précipitation enregistrés par chaque pluviographe pendant chaque durće unitaire d'averse (1 heure par exemple sur les bassins de l'importance de ceux étudiés ici). La précision de cette opération peut être limitée par les défauts de synchronisation des appareils, qui entachent d'erreur la chronologie de la pluie. C'est le hyétogramme moyen, indiquant les tranches horaires des précipitations de l'averse génératrice de crue sur le bassin, qui sera désormais utilisé pour la suite de l'étude (fig. 7).

\section{Analyse de l'hydrogramme de CRue.}

Cette analyse consiste d'une part à séparer les diverses phases d'écoulement (ruissellement direct, ruissellement retardé ou écoulement hypodermique, écoulement souterrain) et d'autre part à étudier leur distribution dans le temps.

\section{a) Séparation des différentes composantes de} l'écoulement :

On peut et doit se demander si l'on a effectivement affaire à ces trois types d'écoulement pour la crue ou sur le bassin étudié et également si la faible importance relative de l'une ou l'autre des phases ne rend pas illusoire la précision du

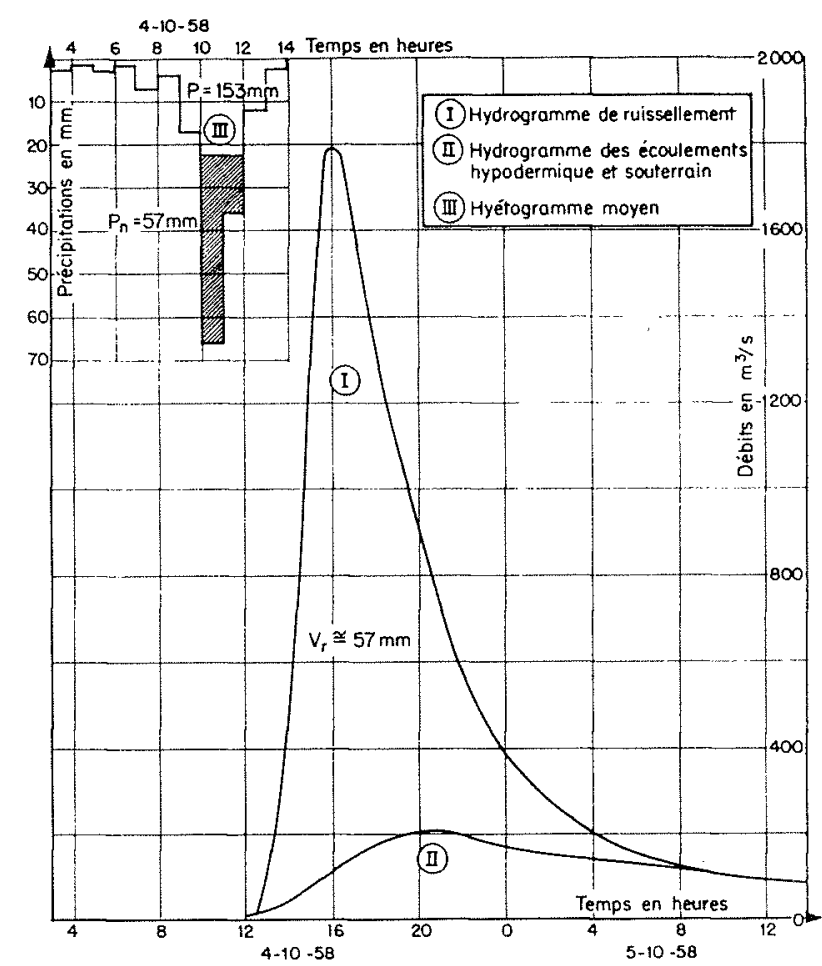

Fre. 7

Hyétogramme moyen et hydrogramme de la crue du Vidourle à Sommières le 4 octobre 1958 (Sur l'hydrogramme, la courbe frontière entre écoulements hypodermique et souterrain n'a pas été tracée.)

tracé de la courbe frontière correspondante sur l'hydrogramme observé.

Dans le cas de la crue du Vidourle à Sommières, le 4 octobre 1958 (fig. 7), provoquée par une averse d'intensité exceptionnelle, la part du ruissellement direct a été prépondérante dans la formation du débit de pointe.

Dans le cas des bassins versants du Massif Central, où les précipitations ont un caractère moins violent, il devient très aléatoire de chercher à distinguer les deux phases du ruissellement et c'est d'ailleurs inutile pour l'application de la méthode de l'hydrogramme unitaire. En effet, lorsque Shermann, vers 1932 , proposa les principes de cette méthode d'analyse, il ne distinguait pas entre ruissellement direct et retarde, et, d'autre part, l'analyse des crues à Alrance a montré que la théorie s'appliquait parfaitement à l'hydrogramme de crue dont on sépare seulement la phase d'écoulement souterrain [2].

Toutefois, il y a intérêt à séparer les deux phases de ruissellement chaque fois que celte opération est possible (crues importantes). En effet, l'hydrogramme de ruissellement direct per. met de mieux déterminer le temps de concentration du bassin, tandis que l'hydrogramme groupant les deux formes de ruissellement a un temps de base plus long et relativement moins 


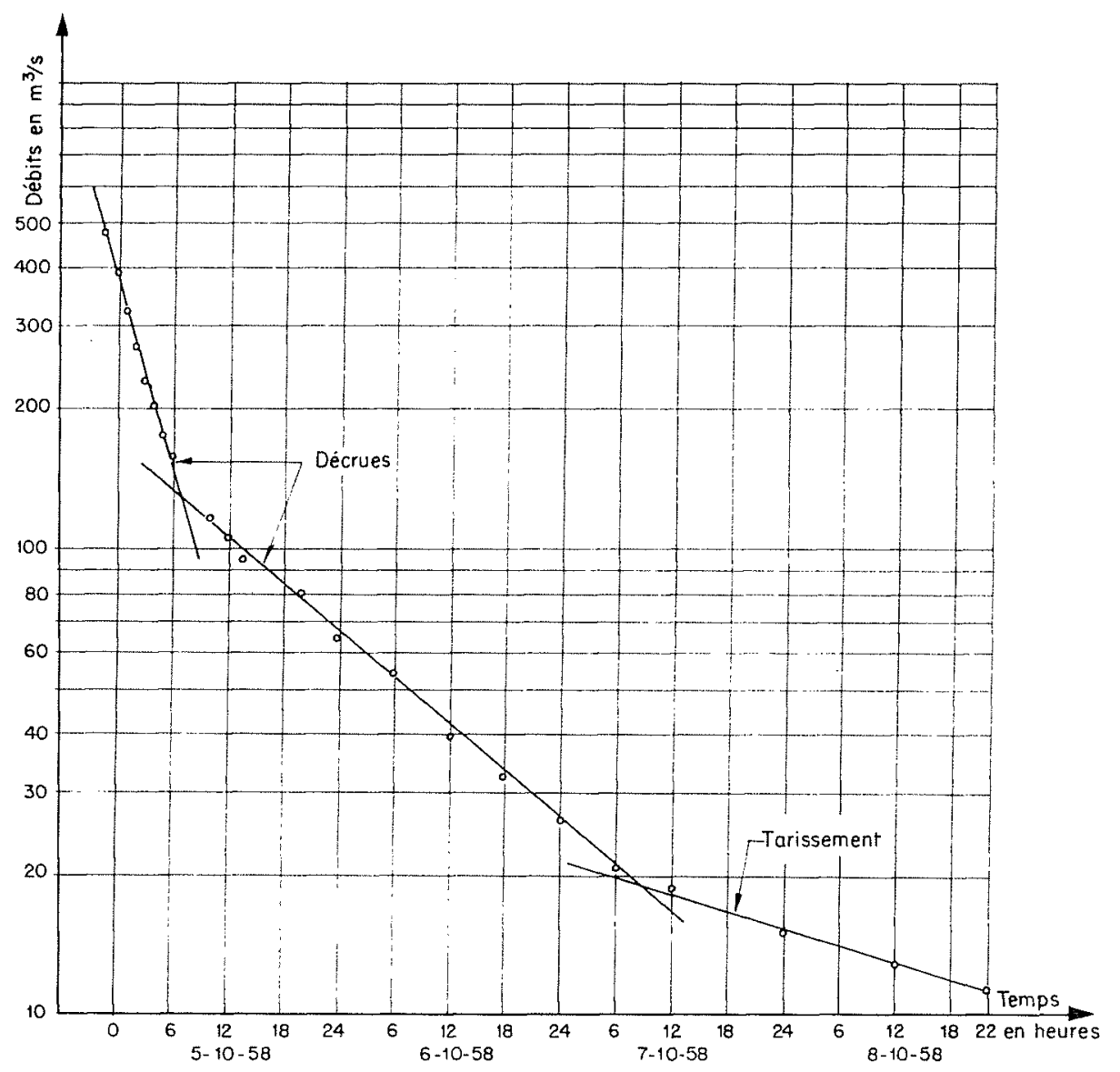

Fig. 8

Etude de la décrue du Vidourle à Sommières du 5 au 8 octobre 1958. (L'échelle des débits est logarithmique.)

bien défini, auquel il n'est pas possible de donner une signification précise.

Les deux méthodes d'analyse d'hydrogrammes ont été utilisées : en trois phases (étude du Vidourle) et en deux (étude des bassins du Massif Central). Dans l'un et l'autre cas, les méthodes de séparation sont analogues et basées sur l'étude des courbes de décrue. On admet couramment que la décrue de chaque phase d'écoulement s'opere suivant une loi de décroissance exponentielle de la forme :

$$
\mathrm{Q}(t)=\mathrm{Q}\left(t_{0}\right) e^{-\alpha\left(t-t_{n}\right)}
$$

L'analyse de la courbe de décrue tracée en coordonnées semi-logarithmicques $(\log Q, t)$ fait apparaître les iustants de fin de décrue des différents types d'écoulement (fig. 8).

On observera que la loi de décrue souterraine (loi de tarissement) n'est valable qu'en l'absence d'évaporation : en réalilé, le coefficient de décroissance $\approx$ varie avec la saison. Ainsi, l'ćtude du tarissement du bassin de la Maronne (fig. 9) a permis de déterminer, par des courbes enveloppes de décrues souterraines, des valeurs moyennes de $\alpha$ significativement différentes :

en été $\quad\left(14<0_{m}<16^{\circ}\right): 10^{6} x=2,05$

en hiver $\left(4<\theta_{m}<5^{\circ}\right): 10^{n} \alpha=1,28$

( $Q$ étant exprimé en $\mathrm{m}^{3} / \mathrm{s}$ et $t$ en secondes).
Le tracé des courbes frontières sur l'hydrogramme étudić est effectué par extrapolation à gauche des courbes de décrue d'écoulement hypodermique ou souterrain, puis complété au jugé. Dans certains cas, l'étude d'hydrogrammes correspondant à des averses n'ayant manifestement pas donné lieu à un ruissellement important permet de définir le temps séparant l'averse des maximums de crues hypodermique ou souterraine et parfois les débits correspondants. C'est finalement l'expérience acquise dans la comparaison des hydrogrammes calculés et observés d'un bassin qui permet d'améliorer le tracé des courbes frontières.

b) Estimation des volumes d'écoulement et bilan global relatif ì une averse :

Dans le cas de la crue du 4 octobre 1958 à Sommières, la séparation des phases d'écoulement de crue conduit aux résultats suivants :

-Volume ruisselé $V_{r}=36 \mathrm{Mm}^{3}$ équivalents à une lame d'eau (pluie nette) $P_{n}=57 \mathrm{~mm}$,

- Coefficient de ruissellement global :

$$
\mathrm{K}_{r}=\mathrm{P}_{n} / \mathrm{P}=57 / 153=37 \% \text {. }
$$

- Le bilan approximatif de l'écoulement de l'averse du 4 octobre s'établit en calculant 


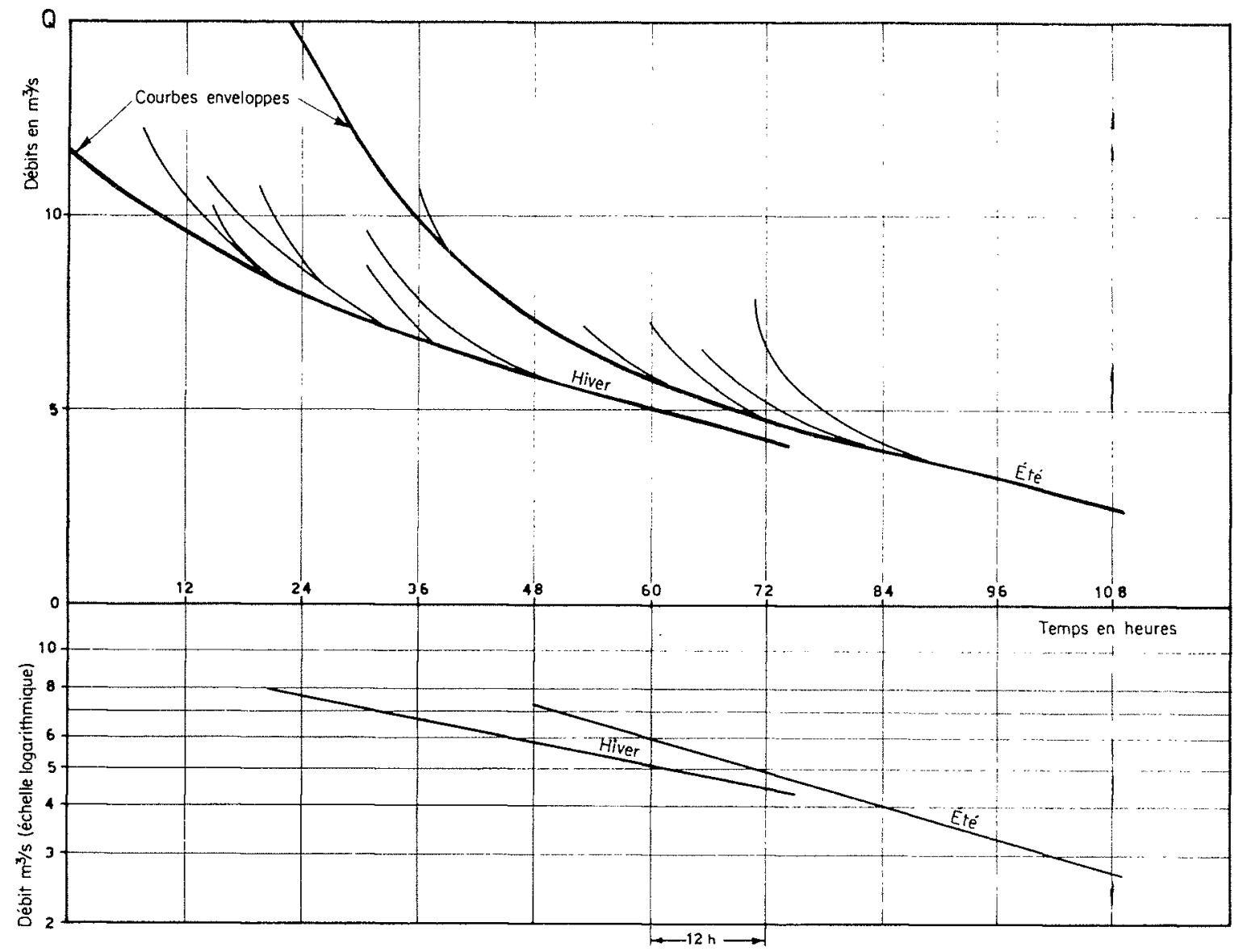

F10. !)

Courbes de tarissement de la Maronne à Sainte-Eulalie (B.V. $113 \mathrm{~km} \mathrm{k}^{2}$ ).

(Les courbes enveloppes ont été tracées en coordonnées semi-logarithmiques dans la partie inferlieure.)

le volume (ou son èquivalent en lame d'eau) écoulé entre le début de la crue et l'instant de réapparition de la valeur du débit de base observé au début de la crue. Il apparaît ainsi que l'averse étudiée $(\mathrm{P}=153 \mathrm{~mm}$ ) a produit $93 \mathrm{~mm}$ d'écoulement, soit un rendement de $60 \%$ : la recharge en humidité du sol et l'évapotranspiration ont absorbé $40 \%$ de la précipitation. Ces chiffres doivent être rapprochés de ceux relatifs aux averses des 29 et 30 septembre 1958 qui ont fourni seulement $17 \%$ de leur volume en écoulement en raison de l'état de sécheresse du bassin [3].

c) Etude de la distribution da volume d'éconlement dans le temps:

On détermine sans difficulté spéciale les temps de réponse du bassin, temps de montée et temps de base de l'hydrogramme de crue [1j. Ces diverses grandeurs varient en fonction des caractéristiques d'averses et interviennent pour fixer la forme de l'hydrogramme relatif à une averse de durée donnce : elles permettenl, en particulier, de déterminer l'heure du débit de pointe correspondant.

Ainsi, sur le bassin de la Maronne orienti ouest-est dans le sens de l'écoulement (fig. 5), le temps de montée de la crue varie du simple au double suivant le déplacement de l'averse d'est en ouest ou d'ouest en est. Dans le cas de la Diège au pont de Loubeix (fig. 2), le lemps de montée $t_{m}$ est lié à la duréc de la pluie $t_{p}$ par la relation linéaire:

$$
t_{m}=\left(t_{p} / 2\right)+8 h
$$

\section{DÉRIVATION D'UN HYDROGRAMME UNITARE $\lambda$

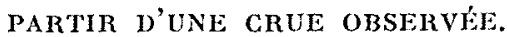

Cette opération est présentée sous la forme simplifiée suivante : définir, à partir de l'hydrogramme de la figure 7 , un hydrogramme unitaire correspondant $\dot{a}$ une averse efficace de 1 heure et à un volume de ruissellement équivalent à $1 \mathrm{~mm}$ de lame d'eau.

a) Le volume de ruissellement de la crue $\mathrm{V}_{\mathrm{r}} \approx 57 \mathrm{~mm}$ de lame d'eau a été produil, 
d'après le hyétogramme moyen, par une fraction d'averse qui a duré 2 heures et fourni $102 \mathrm{~mm}$ de pluie efficace, dont $57 \mathrm{~mm}$ de pluie nette. On a admis pour cela une capacité d'absorption constante sur le bassin pendant cette durée efficace : $\mathrm{C}_{a m}=22,5 \mathrm{~mm} / \mathrm{h}$ (en fait, ce paramètre devrait ètre une fonction décroissante de la durée de la pluie).

b) D'après la théorie de l'hydrogramme unitaire, l'hydrogramme de ruissellement observé peut être considéré comme résultant :

- soit d'une averse de 2 heures avec intensité moyenne de pluie nette:

$$
\mathrm{I}_{n}=57 / 2=28,5 \mathrm{~mm} / \mathrm{h}
$$

- soit de la succession de deux averses unitaires de 1 heure dont les intensités nettes seraient $43 \mathrm{~mm} / \mathrm{h}$ pour la première et $14 \mathrm{~mm} / \mathrm{h}$ pour Ia seconde (fig. 7).

c) La distribution horaire (en \%) du volume ruisselé $V_{r}$ donne une suite de valeurs $v_{i}$ (telles que $\Sigma v_{i}=100$ ) qui constituent dans le premier cas une estimation des coefficients de distribution du ruissellement produit par une averse unitaire de 2 heures.

d) Si l'on considère l'hydrogramme observé comme résultant de la composition de deux hydrogrammes décalés d'une heure, correspondant respectivement à des averses unitaires de 1 heure, d'intensités nettes $43 \mathrm{mmm} / \mathrm{h}$ et $14 \mathrm{~mm} / \mathrm{h}$, les valeurs des coefficients de distribution horaire $u_{i}$ du ruissellement d'une averse de 1 heure, s'obtiennent en résolvant le système d'équations linéaires ex- primant l'égalité des tranches horaires de ruissellement dans les hydrogrammes composé et observé. La méthode est générale et est en principe applicable quelle que soit la complexité de l'averse génératrice et de l'hydrogramme résultant.

Dans tous les cas, le nombre d'équations écrites est supérieur à celui des inconnues $u_{i}$. La théorie de l'hydrogramme unitaire permet d'affirmer la compatibilité du système. La solution devrait donc être indépendante du choix des équations résolvantes: en fait, les imprécisions de tracé de l'hydrogramme de ruissellement, celles de la répartition des intensités nettes entre les averses unitaires, jointes au fait que la linéarité de la relation pluie-ruissellement est seulement une hypothèse, conduisent à des séries de valeurs différentes de coefficients $u_{i}$ selon le choix opéré. On procède alors de manière à réaliser le meilleur ajustement possible pour les valeurs centrales utilisées pour la détermination des débits de pointe.

On a retenu pour l'hydrogramme unitaire du Vidourle à Sommières, dont le temps de concentration est 16 heures, un coefficient maximal de distribution $u_{4}=18 \%$ dans la quatrième heure à partir du début de la crue. Il correspond donc, pour une lame d'eau ruisselée de $1 \mathrm{~mm}$ produite par une averse de 1 heure, le débit de pointe :

$\mathrm{Q}_{\mathrm{M}}=\frac{10^{6} \cdot 0,18}{3600}=50 \mathrm{I} / \mathrm{s} . \mathrm{km}^{2} \sim 31,5 \mathrm{~m}^{3} / \mathrm{s}$

e) En pratique, on se livre rarement au calcul qui vient d'être exposé : on détermine à par-

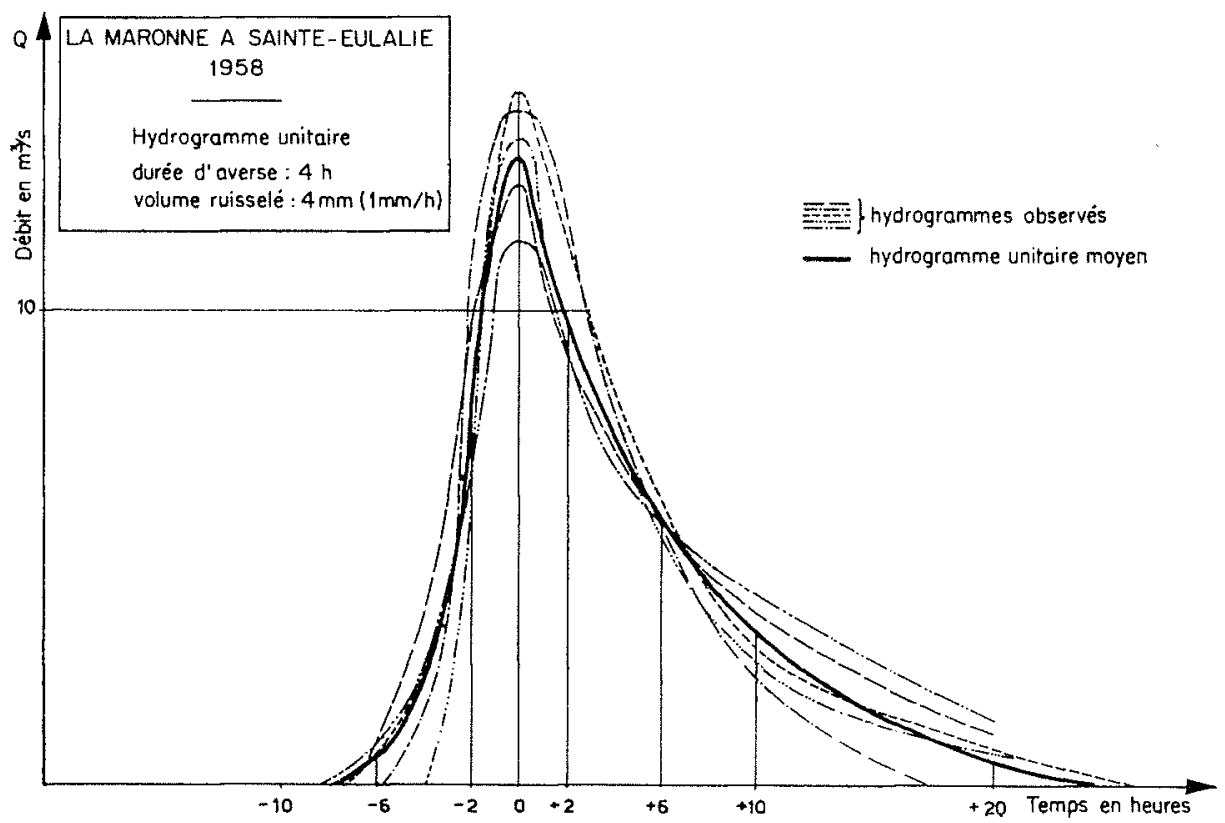

FIG. 10

Hydrogrimme unitaire moyen de la Maronne à Sainte-Eulalie correspondant à une durée d'averse unitaire de 4 heures.

(Cet hydrogramme n'est pas un hydrogramme de ruissellement pur: il intègre tous les types d'écoulement de crue à l'exclusion de l'écoulement souterrain). 


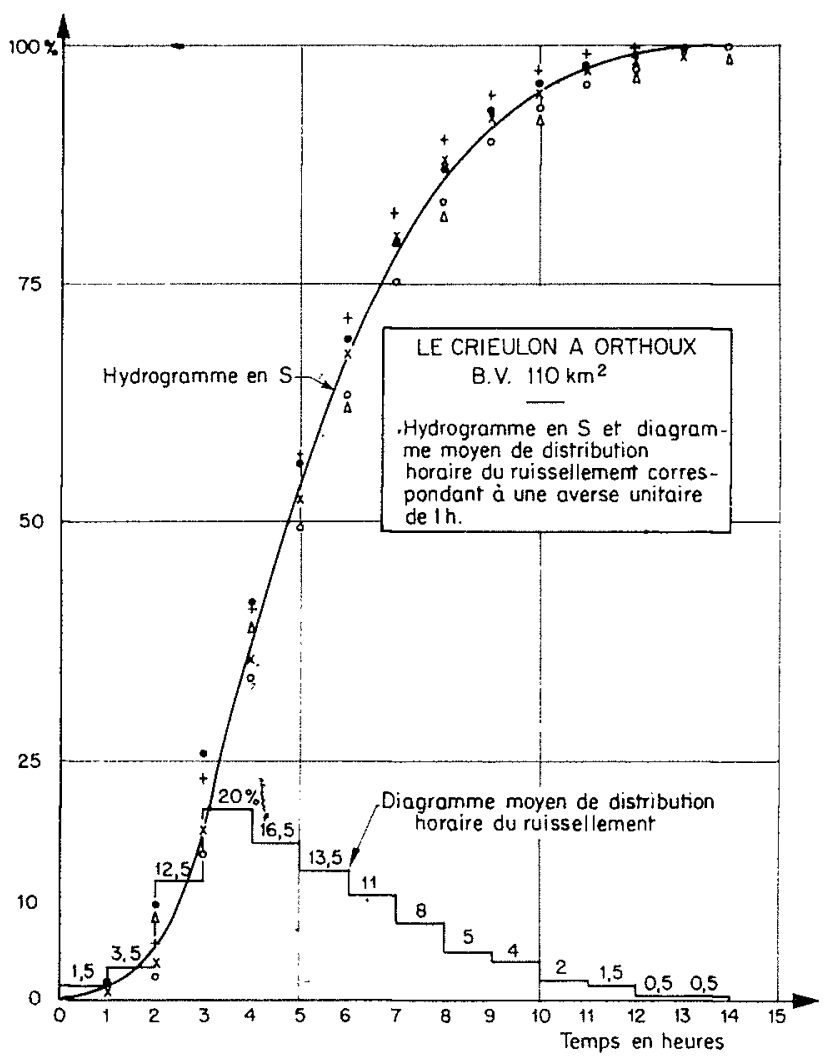

FIG. 11

Hydrogramme en $S$ et diagramme de distribution horaire du ruissellement correspondant à une averse unitaire de $1 \mathrm{~h}$ sur le bassin versant du Crieulon à Orthoux (superficie : $110 \mathrm{~km}^{2}$ ). tir d'averses unitaires de même durée une série d'hydrogrammes unitaires dont on déduit un hydrogramme moyen en amenant en coincidence les temps d'occurrence du débit de pointe (fig. 10). A partir de cet hydrogramme, on compose les hydrogrammes correspondant à diverses durées d'averses nettes et en particulier l'hydrogramme en $S \mathrm{du}$ bassin [1]. Compte tenu des informations sur les caractéristiques des précipitations exceptionnelles, on peut alors définir différentes répartitions en intensité et durée de pluie nette d'averses uniformes susceptibles de produire un débit de pointe de ruissellement donné.

La figure 11 représente l'hydrogramme en $S$ et le diagramme moyen de distribution horaire du ruissellement d'une averse unitaire de 1 heure sur le bassin de Crieulon à $\mathrm{Or}^{*}$ thoux (fig. 4): ils ont été établis à partir d'un certain nombre d'hydrogrammes unitaires et rectifiés d'après les écarts constatés entre hydrogrammes observés et calculés.

\section{Comparaison des CARACTÉristiques D'HYDRO- GRAMMES UNITAIRES DE QUELQUES COURS D'EAU FRANÇAS.}

Les résultats groupés dans le tableau I : temps de réponse, temps de montée, temps de base, temps de concentration et débit de pointe, suffisent à définir convenablement les hydrogram-

TABLEAU I

Caractéristiques d'Hydrogrammes unitaires relatifs à une averse unitaire de durée une heure $\left(\mathrm{V}_{r}=1 \mathrm{~mm}\right)$

\begin{tabular}{|c|c|c|c|c|c|c|c|c|c|c|}
\hline \multirow[t]{2}{*}{ Bassin versant } & \multirow{2}{*}{$\begin{array}{l}\begin{array}{l}\text { Super- } \\
\text { ficie } \\
\text { en } \mathbf{k m}^{2}\end{array} \\
\text { A }\end{array}$} & \multicolumn{3}{|c|}{ Altitudes (en m) } & \multirow{2}{*}{$\begin{array}{c}\text { Temps } \\
\text { réponse } \\
\text { "lag" } \\
t_{n}\end{array}$} & \multirow{2}{*}{$\begin{array}{c}\text { Temps } \\
\text { de montée } \\
t_{m}\end{array}$} & \multirow[t]{2}{*}{$\begin{array}{l}\text { Temps de } \\
\text { hase }\end{array}$} & \multirow{2}{*}{$\begin{array}{c}\text { Temps } \\
\text { de concen- } \\
\text { tration } \\
\text { t. }\end{array}$} & \multicolumn{2}{|c|}{$\begin{array}{l}\text { Débit } \\
\text { de pointe } \\
\text { de l'hydr. } \\
\text { unitaire }\end{array}$} \\
\hline & & Maxi. & Moy. & Mini. & & & & & $\mathrm{m}^{3} / \mathrm{s}$ & 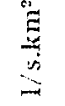 \\
\hline Alrance $\ldots \ldots \ldots \ldots$ & 3,15 & 960 & 875 & 770 & $1 \mathrm{~h}$ & $1 \mathrm{~h} 10^{\circ}$ & $12 \mathrm{~h}$ & $3 \mathrm{~h}$ & 0,375 & 119 \\
\hline Vidourle à Saint-Hippolyte. & 35,5 & 975 & 421 & 165 & 2 à $3 \mathrm{~h}$ & $1 \mathrm{~h} 30^{\prime}$ & $20 \mathrm{~h}$ & 6 à $8 \mathrm{~h}$ & 2,65 & 75 \\
\hline Crieulon à Orthoux....... & 110 & 890 & 139 & 54 & 7 à $8 \mathrm{~h}$ & $1 \mathrm{~h} 30$ à $2 \mathrm{~h}$ & 22 à $24 \mathrm{~h}$ & 12 a $14 \mathrm{~h}$ & 6,0 & 55 \\
\hline Maronne à Sainte-Eulalie.. & 113 & 1741 & 985 & 570 & 6 à $8 \mathrm{~h}$ & $2 \mathrm{~h}$ & $26 \mathrm{~h}$ & & 4.5 & 40 \\
\hline Diège à Pont du Loubeix.. & 225 & 954 & 775 & 635 & 8 à $10 \mathrm{~h}$ & $8 \mathrm{~h}$ & $30 \mathrm{~h}$ & & 7,0 & 31 \\
\hline Bès à Saint-Juéry....... & 283 & 1471 & 1194 & 917 & $10 \mathrm{~h}$ & $5 \mathrm{~h}$ & $32 \mathrm{~h}$ & & 8,3 & 29 \\
\hline Lander à Saint-Georges... & 310 & 1497 & 989 & 749 & 6 à $7 \mathrm{~h}$ & 3 à 4 h & $32 \mathrm{~h}$ & $16 \mathrm{~h}$ & 13,0 & 42 \\
\hline Vidourle à Sommières. & 630 & 975 & 174 & 21 & $|8 \dot{a} 10 h|$ & $3 \mathrm{a} 4 \mathrm{~h}$ & $36 \mathrm{~h}$ & 16 à $18 \mathrm{~h}$ & 31,5 & 50 \\
\hline
\end{tabular}


mes unitaires des bassins versants étudiés.

Les hydrogrammes unitaires relatifs au $\mathrm{Vi}$ dourle et au Cirieulon concernent le seul ruissellement direct; les autres intègrent également le ruissellement retardé ou hypodermique.

L'hydrogrramme unitaire étant essentiellement une des caractéristiques d'un bassin versant, l'examen des données contenues dans le tableau I permet d'entrevoir certaines liaisons entre les grandeurs représentatives des bassins et les hydrogrammes correspondants :

- le temps de réponse croît avec l'importance du bassin versant et varie en raison inverse de sa pente moyenne. Il présente également des variations selon le sens de déplacement de l'averse, jusqu'à diminuer de moitié dans le cas d'une progression de la pluie de l'amont vers l'aval (Vidourle, Maronne);

- le temps de montée est affecté par le déplacement de l'averse (Maronne), mais il est surtout sensible à la pente moyenne du bassin (Diège, Bès);

- les temps de base reportés sur le tableau sont ceux d'hydrogrammes comprenant l'écoulement hypodermique : leurs valeurs sont donc supérieures à celles définies par la relation $\mathrm{T}_{b}=t_{c}+t_{c}$ (temps de base de l'hydrogramme de ruissellement $=$ temps de concentration + durée de pluie nette). Certains temps de concentration sont ici assez mal définis, en raison du petit nombre de crues analysées;

- les débits spécifiques de pointe décroissent rapidement lorsque la superficie du bassin croit. La pente moyenne, la forme du bassin, sa couverture végétale, sa nature géologique et pédologique, la violence des précipitations (part prépondérante de ruissellement direct, réduction du temps de base) les affectent également : autant de relations à préciser au fur et à mesure de l'extension des recherches d'hydrogrammes unitaires sur des bassins variés.

\section{B. - Application des hydrogrammes unitaires à la synthèse d'hydrogrammes d'écou- lement}

Les analyses précédentes n'ont d'intérêt réel que si elles contribuent à la mise au point de méthodes permettant de déterminer à l'avance ou de reconstituer l'hydrogramme d'écoulement d'une averse. En ce domaine, les travaux n'ont pas été suffisamment systématiques pour que l'on puisse présenter des résultats cohérents d'application d'hydrogrammes unitaires à la prévision des débits : on a toutefois vérifié que les hydrogrammes types dont on dispose permettent de représenter correctement la répartition dans le temps des débits provenant d'un épisode pluvieux complexe (fig. 12).

D'ailleurs le problème véritable est d'abord celui de la détermination du volume d'écoulement produit par l'averse : l'hydrogramme unitaire permettant ensuite d'étudier la répartition de ce volume dans le temps.

Les problèmes à résoudre concernent donc surtout les coefficients de ruissellement et d'écoulement des averses :

1. La détermination de la pluie efficace et de la pluie nette nécessite une étude de la capacitó d'absorption du bassin en fonction du montant et de la durée de l'averse et de divers autres facteurs (écart à la pluie antérieure, etc.).

2. Dans le cas de bassins du Massif Central, soumis à des régimes hydrologiques assez réguliers, le coefficient de ruissellement paraît pou-

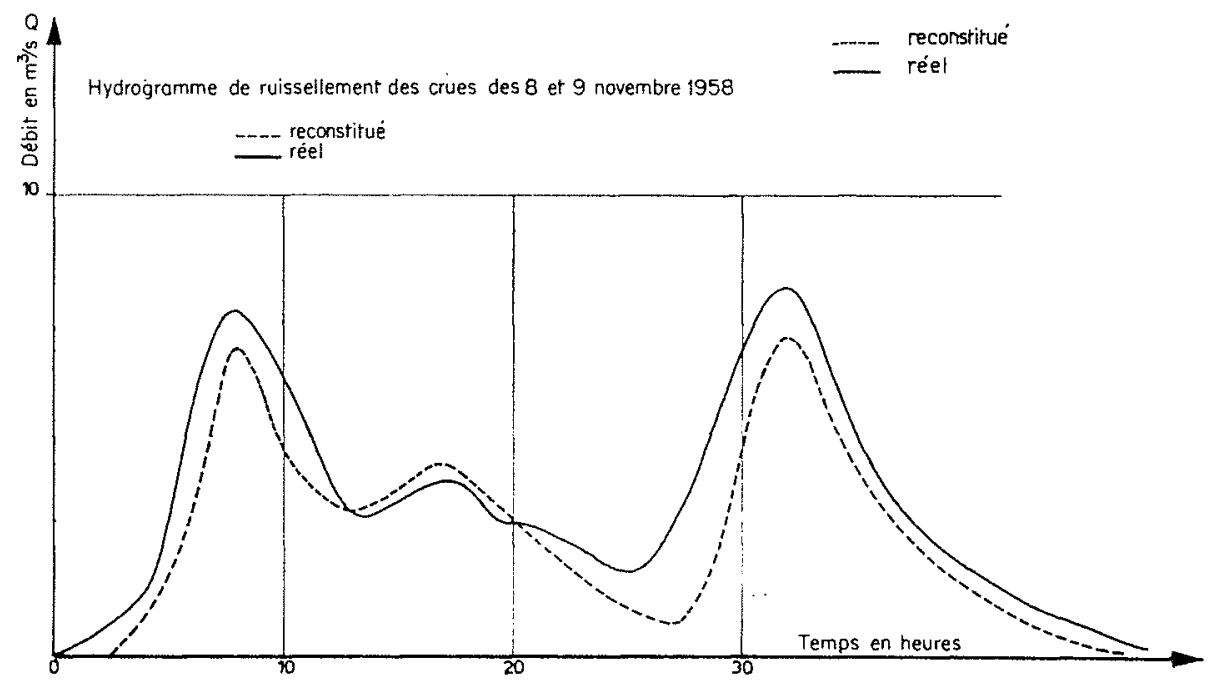

Fig. 12

Reconstitution de la Maronne à Sainte-Eulalie. 
voir être lié assez simplement à un paramètre l'eprésentant l'état dú bassin (débit de base par exemple) et à un paramètre représentant la précipitation qu'il reçoit [2].

3. Les coefficients de ruissellement des crues analysées dépassent rarement $20 \%$. La plus grande partie des eaux météoriques est donc évacuée par l'écoulement souterrain (sauf le cas de crues exceptionnelles telles que celle du 4-101958 à Sommières) : d'où l'importance de la recherche des lois de cette phase de l'écoulement sur les bassins où la prévision des débits doit porter non seulement sur les débits de pointe de crues, mais être continue et englober la totalité des apports d'écoulement.

\section{CONCLUSION}

La recherche d'hydrogrammes unitaires de cours d'eau français en est à ses débuts : la précision des résultats ira s'améliorant dans la mesure où des études systématiques seront poursuivies sur des bassins versants de types divers.

Certains obstacles à la mise en œuvre de cette technique sont dus à des causes susceptibles d'être éliminées par des équipements convenables (toutes les questions relatives à la densité et à la précision des observations), d'autres sont liés aux caractéristiques des bassins versants qui ne répondent pas aux conditions théoriques d'application de la méthode.

Ainsi l'hétérogénéité de répartition de la pluie est généralement d'autant plus accusée que la superficie du bassin versant est plus grande : il sera donc nécessaire d'étudier des bassins élémentaires à caractéristiques relativement homogènes ou bien de travailler à une échelle moins fine (précipitations de $6 \mathrm{~h}, 12 \mathrm{~h}, 24 \mathrm{~h}$ ). Il reste que la méthode devra toujours être adaptée par l'utilisateur aux données dont il dispose et aux conditions de ses problèmes.

Enfin, la recherche de l'hydrogramme unitaire n'est qu'une phase de l'étude des lois d'écoule. ment d'un bassin versant dont la connaissance expérimentale ou statistique permet seule de répondre de façon satisfaisante aux problèmes de la prévision des débits des cours d'eau.

RÉFEIRENCES BIBLIOGRAPHIQULS

11] G. Remenienas. - L'Hydrologie de l'Ingénieur. Eyrolles, Paris, 1960. Chap. VIII : Analyse et prédétermination de l'hydrogramme afférent à une averse donnée, pp. 309-376.

[2] P. Cappus. - Bassin expérimental d'Alrance : étude des lois de l'écoulement, application au calcul et à la prévision des débits. La Houille Blanche, $\mathrm{n}^{\circ} \mathrm{A}, 1960$, pp. 493-520, 12 fig.

[3] J. JacQuer. - Les crues d'automne 1958 sur le Vidourle. La Houille Blanche, $\mathrm{n}^{\circ}$ A, 1959, pp. 66-82, 6 fig.

\section{I S C US I O N}

Président : M. DeYmé

\footnotetext{
Monsieur le Président remercie M. JACQUeT d'avoir montré, en particulier, comment on déduit l'hydrogramme unitaire du diagramme de distribution des crues observées.

Sur sa demande, M. JacQuer précise que les temps de base indiqués sur le tableau annexé à sa communicalion sont ceux de l'hydrogramme de ruissellement global incluant l'écoulement hypodermique: les deux phases du ruissellement (direct et retardé) n'ayant pu être séparées pour certains bassins étudiés. Ce temps de base est beaucoup plus important que celui de l'hydrogramme
}

de ruissellement direct et sa détermination est moins précise.

M. Remenieras confirme la variabilite du lemps de base de l'écoulement hypodermique qui n'a d'autre signification physique que celle que l'on peut difinir sur l'hydrogramme lui-même. Il est donc peu ŝ̂r de calculer des débits de crue sur des hydrogrammes intégrant les ruissellements direct et relardé.

M. le Président fait observer que les temps de hase indiqués par M. Jacever sont considérables ef bien 
supérieurs à la somme du temps de concentration et de la durée de l'averse puisqu'ils atteignent deux fois le temps de concentration.

M. Rodrer indique qu'il a constaté sur des hydrogrammes des temps de montée hypodermique doubles des temps de montée du ruissellement superficiel. Par ailleurs, la variabilité des temps de montée croît encore quand on passe de l'écoulement hypodermique à l'écoulement souterrain.

M. Fenry considère qu'en pratique, il importe de connaître le volume d'eau écoulé pendant un temps bien défini, fixé conventionnellement une fois pour toutes, qui permettra de confronter les résultats des prévisions et les observations effectives.

M. Rodren estime que l'on peut accepter une assez grande indétermination sur le temps de base pour le calcul des débits de crue : il suffit de mesurer à 5 on $10 \%$ près le volume de ruissellement sur l'hydrogramme. Le paramètre temporel important est le temps limite deffinissant l'averse unitaire, temps qu'il ne faut pas dépasser.

M. REMENIERAS fait remarquer que le débit maximum, auquel on s'interesse le plus en pratique, est lié au volume de ruissellement ou d'éconlement de crue par un cocfficient de répartition : une erreur sur le temps de hase se répereute sur ce coefficient et affecte donc lo (lébit maximum calculé.

M. Fenry estime que le temps de base doit être défini par des règles fixes : dans un but d'homogénéité, il sera pris égal, par exemple, à la durée de la pluic augmentéc d'une constante.

M. Remenienas pense qu'il est bon de se rattacher pour cela à un modèle physique, mais l'hydrogramme unitaire ne constitue un modèle satisfaisant que pour l'étude de la phase du ruissellement superficiel.

M. Guillot, qui désire apporter le point de vue d'un utilisateur (E.D.F. - Production Hydraulique), pense, en substance, que la théorie de l'hydrogramme unitaire fait bien comprendre le mécanisme de formation de la crue, mais son application ne fournit que la chronologie du phénomène "courbe en cloche dissymétrique". Ce dont l'utilisateur a besoin, c'est de savoir à l'avance quelle est la proportion du volume de pluie qui va s'écouler dans un délai de l'ordre de 48 heures. L'étude systématique de tous les paramètres susceptibles de déterminer le coefficient d'écoulement s'impose done : l'état de la végétation (lié à la saison), l'humidité du sol, - plus ou moins bien reprèsentée par un index des pluies antérieures ou le débit de la rivière avant la crue - et l'intensité de l'averse; encore que, pour ce dernier paramètre, dans de nombreux bassins en France, M. Guillot croit que l'intensité de pluie due à un passage cyclonique - front chaud plus front froid, ou front froid seul, de durće totale 6 à 12 heures atteint rarement le seuil qui provoque. le ruissellement et qu'alors le paramètre "intensité de pluie» n'a, sans doute, qu'me influence secondaire. Il faut s'efforcer d'établir, le plus tôt possible et pour plusieurs bassins, un catalogue de plusieurs centaines de crues courantes, correspondant chacune au passage d'une perturbation. Seules ces données - et non des raffinements de forme d'hydrogrammes unitaires - - permettront les études de cowrélation indispensables pour prévoir le coefficient d'écoulement d'après les conditions antérieures d'une averse.

M. le Président indique que les orateurs qui se sont succédés, en particulier M. Rodier, se sont efforcés de répondre à cette question.

M. Ropmen estime que l'hydrogramme unitaire est d'un tris grand interèt pour le calcul des crues exceptionnelles dues principalement au ruissellement. Deux facteurs interviennent dans ce calcul : le volume écoulé et la variation du débit écoulé (forme de l'hydrogramme). La précision de ce dernier facteur ne doit pas ĉtre négligée sous le prétexte que l'on connaît assez mal le premier : les deux sont nécessaires à la détermination du débit de pointe avec la précision optimale. En pratique, sur un bassin expérimental, l'étude de la forme de l'hydrogramme amène à étudier tous les paramètres liés au cocfficient d'écoulement qui intéresse M. Guillor. Il est évident, par contre, que l'hydrogramme unitaire n'est d'aucune utilité pour l'étude des petites pluies qui ne ruissellent pas.

M. Nonmaxo demande si l'utilisation de l'hydrogramme unitaire pour la prévision des crues exceptionnelles est possible it partir d'une statistique des pluies journalières. La duréc de la pluie unitaire sur les petits bassins étudiés par M. JACQUET est en général de une heure, l'étude statistique des crues suppose donc une étude statistique préalable portant sur des hyétogrammes ou des données pluviométriques horaires qui ne sont généralement pas fournies par les Services Météorologiques. Il scmblerait done que le manque d'informations pluviométriques adéquates limite considérablement l'intérêt de la méthode pour. l'étude des crues exceptionnelles de petits bassins.

M. JACQUET rípond que le calcul des crues exceptionnelles par li méthode de l'hydrogramme unitaire nécessite effectivement l'étude des intensités d'averses, qu'il n'est pas possible de déduire d'observations pluviométriques journalières dans le cas de petits bassins. On dispose en France d'assez nombreuses séries de hyétogrammes d'importances diverses, à partir desquelles il serait souhaitable de constituer par région des statistiques d'averses par intensités et durées analogues à celles effectuées par M. Grisollet pour Paris et M. Godard pour Montpellier sur des périodes de trente ans et plus. De toute facon, il est à peu près exclu que l'hydrologue dispose a priori d'une statistique des intensités de pluies au point où il opère: il lui faut en général, utiliser les caractéristiques d'averses exceptionnelles définies sur une région homologue à la zone étudiée, en opérant les ajustements convenables d'après les résultats de quelques campagnes de mesures.

M. Remenieras fait observer que l'intensité de pluie à prendre en considération est liée à la durée de pluie unitaire, elle-même liée à l'ordre de grandeur du temps de concentration : plus le bassin est petit, plus son temps de concentration est réduit et plus fine sera l'étude de l'intensité de la pluie. Pour des bassins de plusieurs milliers de $\mathrm{km}^{2}$, la considération des pluies journalières suffit. Par contre, sur un bassin de quelques $\mathrm{km}^{2}$, une intensité de $1 \mathrm{~mm} / \mathrm{mn}$, qui n'est pas très exceptionnelle, représente un débit de ruissellement de $16,6 \mathrm{~m}^{3} / \mathrm{s} . \mathrm{km}^{2}$. Si Ic temps de concentration d'un tel bassin est de l'ordre de l'heure, on peut s'attendre à voir des débits de erue de $10 \dot{\text { à }} 20 \mathrm{~m}^{3} / \mathrm{s} . \mathrm{km}^{2}$ produits par une averse in tense de une heure: on l'a constaté avec surprise sur des déversoirs de bassins de $1 \mathrm{~km}^{2}$, deux ans après leur construction.

M. Remenieras souligne que l'intensité prise en compte est évidemment celle de la pluie ruisselée. Dans l'exemple (Vidourle) présenté par M. JacQueT, cette pluie a duré $2 \mathrm{~h}$ sur un hassin de $630 \mathrm{~km}^{2}$; cette durée étant très inférieure an temps de concentration, on n'al pas observé les débits spécifiques énormes qui viennent d'être évoqués. Mais cettc durée de pluie nette augmontant, on verrait croître rapidement les débits spécifiques dont la méthode de l'hydrogramme unitaire permet ici de calculer les ordres de grandeur.

M. Cumrot indique que, sur le Vidourle, la crue du 4-10-1958 a été aggravée par rapport a celle du 30-9-1958 du fait même de celle-ci, alors que les intensités pluviales étaient du même ordre.

M. Jacouer rappelle que le volume d'écoulement de 
la crue du 4 octobre représente $60 \%$ du volume de la précipitation, alors que celui du 30 septembre s'élèv a $17 \%$ seulement, La disparité d'importance des deux crues citées a une autre cause : la répartition spatiale de l'averse, bien centrée sur le bassin le 4-10-1958, alors qu'elle en a touché seulement la partie $\mathrm{N}-\mathrm{O}$ lc 30 septembre 1958.

M. Rodıer cite le cas particulièrement caractéristiquc d'un bassin assez perméable où, en trois ans, cing pluies de même intensité $(100 \mathrm{~mm} / \mathrm{h})$ et de durées du mème ordre (deux, trois ou quatre heures) ont donné des volumes ruisselés variant de un à dix, suivant qu'elles tombaient au début ou à la fin de la saison des pluies.

M. FRRTY demande si la définition du rendement d'une nverse à partir du volume d'écoulement calculé en prolongeant à l'infini les courbes de tarissement, avant et après la clue, ne conduit pas à créditer les averses de rendement de même ordre, quel que soit l'état de saturation du terrain.

M. Remenieras fait remarquer qu'effectivement cette définition n'est pas bonne et qu'il est préférable dc rechercher - en plus du coefficient de ruissellement un coefficient d'écoulement calculé sur une période de temps déterminée, par exemple entre l'instant de début de la crue et celui oì réapparaît, après la crue, la valeur de débit de base observé au début de la crue.

M. Jacouer indique que la loi de tarissement de forme exponentielle n'est valable que si les nappes phréatiques $d u$ bassin sont profondes et peu affectées par les phénomènes d'évapotranspiration. Dans le cas jrécis du Vidourle, les courbes de tarissement varient d'une crue à l'autre, ce qui montre la sensibilité des nappes aux variations de l'évaporation dont il faudrait tenir compte en prolongeant ces courbes.

M. Remenieras répète que l'on obtiendrait en effet un meilleur invariant en matière de tarissement en ajoutant à la courbe observée un correctif prenant en compte l'évaporation.

M. le Président souligne que M. JAcQuer a déterminé un coefficient de ruissellement de $37 \%$ pour l'averse du 4-10-1958 sur le Vidourle, coefficient qui est loin de l'unité pour une crue pourtant assez exceptionnelle

M. Rementeras attire l'attention sur les diverses défnitions du «coefficient de ruissellement». On dit en effet couramment que les coefficients de ruissellement des grandes crues sont de 0,9 : les coefficients de ruis- sellement global $K_{r}$, déterminés par $M$. Ropres et M. JACQUET, ont du mal à atteindre ou dépasser 0,5 .

$$
* * *
$$

Pour en revenir à la question des erreurs d'ordres de grandeur différents soulevée par M. Ropien à propos du volume d'écoulement et de la forme de l'hydrogramme, M. FERry croit qu'en effet, il ne faut pas tolérer d'erreur systématique, si faible soit-elle. Par contre, les erreurs aléatoires se compensent et disparaissent totalement ou en grande partie : si l'erreur incompressible sur l'un des facteurs est de 1 , le fait de négliger une erreur de l'ordre de $1 / 3$ sur l'autre n'augmente que de $5 \%$ l'erreur globale calculée suivant la méthode de Gauss. C'est de ce point de vue que l'on peut dire que la précision du second facteur ne vaut pas la peine d'être améliorée.

$$
\text { 䑩 }
$$

A la suite de lintervention de M. Nomaxis, M. Romin a tenu à préciser l'intérêt pratique de la methode do l'hydrogramme unitaire pour l'étude des crues exceptionnelles sur de petits bassins en Afrique par la note suivante :

\& 1. Dans tous les pays africains où nous avons exercé notre activité, nous avons, sans difficulté, calculé l'intensité horaire des pluies à partir de l'ensemble des relevés des pluviomètres et des pluviographes.

$2^{\circ}$ L'étude des crues exceptionnelles est très importante sur les petits bassins pour la réalisation de ponts et de petits barrages et, seule, la méthode des hydrogrammes permet ce calcul. Plusicurs dizaines de déversoirs ont cédé, ces dernières aumées, cn Mauritanie et en Haute-Volta, par suite du manque de données sur les crues exceptionnelles, et c'est à la suite de ces désastres qu'on a dû développer les études de bassins expérimentaux pour en éviter le retour.

$3^{\circ}$ Même dans le cas de bassins de moyenne importance, ces études fournissent des indices précieux qui peuvent tranquilliser le projeteur, ou, au contraire, lui laisser soupçonner des phénomènes que ne peuvent pas indiquer des relevés trop peu nombreux.

Nous ajouterons, pour souligner l'intérèt pratique de ce procédé, que $90 \%$ de nos études sur bassins expérimentaux ont été payés par des Services Techniques pour en obtenir, en échange, des chiffres précis en vue cle réalisations immédiates. 》

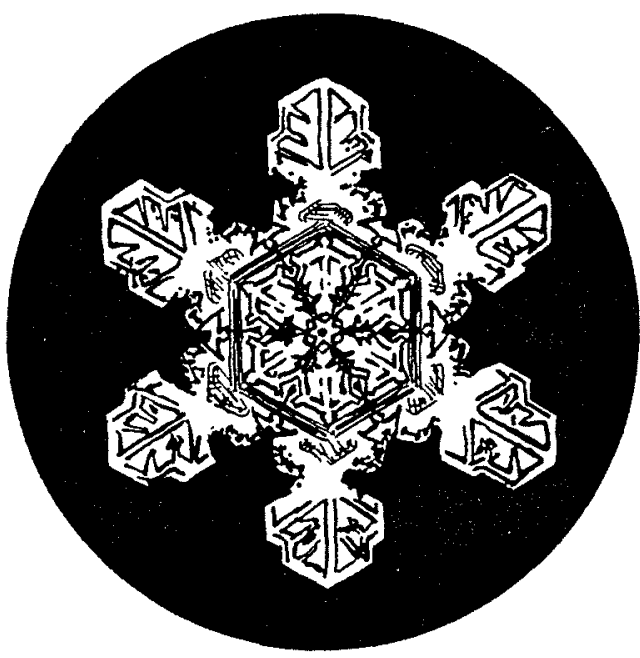

